\title{
The Evolution of Central Stars of Planetary Nebulae
}

\author{
Falk Herwig $^{1}$, Bernd Freytag ${ }^{1,2}$ and Klaus Werner ${ }^{3}$ \\ ${ }^{1}$ Theoretical Astrophysics Group, Los Alamos National Laboratory, Los Alamos, \\ NM 87545, USA \\ email: fherwig@lanl.gov \\ ${ }^{2}$ Department of Astronomy and Space Physics at Uppsala University, Sweden \\ email: bf@astro.uu.se \\ ${ }^{3}$ Institut für Astronomie und Astrophysik, Universität Tübingen, Sand 1, D-72076 \\ Tübingen, Germany \\ email: werner@astro.uni-tuebingen.de
}

\begin{abstract}
The evolution of central stars of planetary nebulae can proceed in several distinct ways, either leading to H-deficiency or to H-normal surface composition. Several new simulations of the evolution channels that lead to H-deficiency are now available, mainly the born-again scenarios that are triggered by a He-shell flash during the hot pre-white dwarf evolution phase. A realistic AGB progenitor evolution is important for correct HRD tracks, that allow mass determinations. New hydrodynamic simulations of He-shell flash convection including cases with $\mathrm{H}$-ingestion are now performed, and allow a determination of the convective extra-mixing efficiency. This has direct consequences for the intershell abundance distribution of AGB stars that can be observed in the H-deficient CSPN.
\end{abstract}

Keywords. stars: AGB and post-AGB, stars: evolution, hydrodynamics

\section{Introduction}

The evolution of the central stars of planetary nebulae (CSPN) is closely connected to the evolution of the progenitor phase, the Asymptotic Giant Branch (AGB) stars, and has observable effects for the CSPN progeny, the WD stars. The AGB evolution phase is the result of previous main-sequence and horizontal-branch evolution of lowand intermediate mass stars. An updated classification of mass ranges involving AGB stars has been recently suggested by Herwig (2005, Fig. 2). Accordingly, low-mass stars are those below an initial mass of $\approx 1.8 \mathrm{M}_{\odot}$ that ignite a He-core flash at the tip of the RGB, while intermediate mass stars are those initially more massive than $\approx 1.8 \mathrm{M}_{\odot}$ but less massive than required to explode as a core-collapse supernova. The dividing mass at $\approx 10 \mathrm{M}_{\odot}$ separates those super-AGB stars that form ONe-white dwarfs and those that ignite as core collapse supernova (e.g. García-Berro \& Iben 1994). However, quantitatively the fraction of AGB stars that explode as core-collapse supernova is still very uncertain, largely due to uncertainties in super-AGB mass loss, and the physics of mixing and burning, e.g. during dredge-up and hot-bottom burning (see below). For AGB stars it is useful to use the limiting mass for hot-bottom burning (HBB) as a dividing line for low-mass and massive AGB stars. Hot-bottom burning transforms dredged up C into $\mathrm{N}$ and prevents the formation of C-rich composition (e.g. Scalo et al. 1975; Lattanzio 1992). HBB has therefore an important influence on the evolution of an AGB star, and the composition of the ejecta that later form the planetary nebula. AGB stars that are massive enough to ignite carbon burning are the super-AGB stars. 


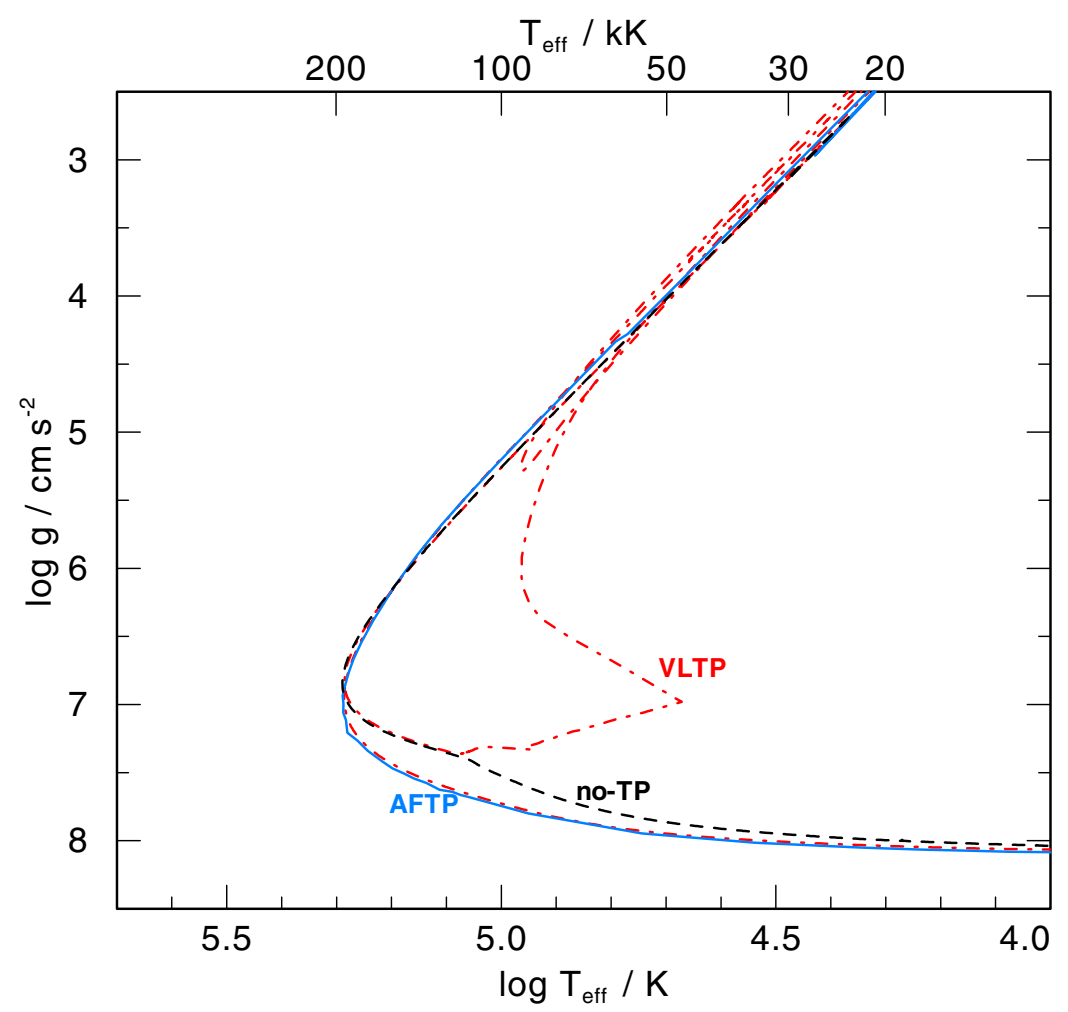

Figure 1. Comparison of three different evolution channels in the $g-T_{\text {eff }}-$ plane for a $0.604 \mathrm{M}_{\odot}$ post-AGB star that had a main-sequence mass of $2 \mathrm{M}_{\odot}$ and evolved through about a dozen thermal pulses with dredge-up. This figure originally appeared in the Publications of the Astronomical Society of the Pacific (Werner \& Herwig 2006). Copyright 2005, Astronomical Society of the Pacific; reproduced with permission of the Editors.

Conceptually, the evolution of AGB stars is well understood, at least at solar and modest metal deficiency, like in the Magellanic Clouds. The review by Iben \& Renzini (1983) describes the basic properties of AGB stars: thermal pulses, the necessity of the third dredge-up, the effect of mass loss and the potential for important nucleosynthesis. More recent reviews by Lattanzio \& Boothroyd (1997) and Blöcker (1999) include accounts of hot-bottom burning, and an improved quantitative description of AGB evolution. Dedicated reviews were written on mass loss (Willson 2000), the $s$ process (Busso et al. 1999; Meyer 1994) and the cool post-AGB stars that allow important observations concerning the $s$ process (van Winckel 2003).

Unfortunately, major unresolved issues remain, that severely limit the predictive quality of current AGB modeling (Herwig 2005). The issues concern the mass-loss history, the nature and importance of hydrodynamic mixing, including the physics of mixing at the bottom of the envelope convection zone that seems to be critical for the third dredge-up, and for formation of the assumed ${ }^{13} \mathrm{C}$-pocket. Another problem is the rather poorly known effect of rotation and magnetic fields on the evolution, nucleosynthesis and formation of asymmetries of bi-polar structure in proto-planetary nebulae. All these problems are amplified for stars at very low metallicity, partly because less observational checks are available.

The evolution of CSPN (described in Sect. 2) depends sensitively on the progenitor AGB evolution. In particular, the observed abundances of the H-deficient CSPN are 
linked to mixing processes in the He-shell flash of the AGB phase, and some new results in this area are presented in Sect. 3.

\section{Evolution of CSPN}

We have now modern stellar evolution models of several distinct channels of post-AGB evolution, and through the CSPN phase (Werner \& Herwig 2006). These channels are characterized by the thermal pulse cycle phase at which they depart from the AGB. The most common is the undisturbed evolution off the AGB, through the CSPN phase and onto the cooling track of white dwarfs. These CSPN should show a surface abundance pattern that reflects the former AGB envelope including enrichment of that envelope through dredge-up (Napiwotzki 1999).

The possibility of a born-again evolution was recognized early, by Schönberner (1983) and Iben et al. (1983). Several more recent calculations are now available (Herwig et al. 1999; Lawlor \& MacDonald 2003), including a new grid of H-deficient CSPN tracks based on the born-again scenario by Miller Bertolami et al. (2006). Two flavours of the born-again evolution are now distinguished: in the very late thermal pulse (VLTP) the remaining small amount of $\mathrm{H}$ in the surface layers is convectively mixed into the He-shell flash convection, while in the late thermal pulse (LTP) this does not happen (Blöcker 2001; Herwig 2001b). It should be noted that the physics of convective-reactive mixing which characterizes the VLTP is not well understood, as emphasized by Herwig (2001a). Currently, efforts are underway at Los Alamos and elsewhere to study the hydrodynamic aspects of this problem. The simulations discussed in the following section are a first step into that direction.

According to their different nucleosynthetic and mixing evolution the VLTP and LTP cases will produce CSPN with observationally distinguishable properties. For example, while Sakurai's object and V605 Aq are now considered the result of a VLTP evolution, FG Sge is the result of a LTP evolution, and the arguments in the literature leading to this conclusion have been reviewed recently by Werner \& Herwig (2006).

In addition to the two born-again channels there may be a possibility for a H-deficient, but not $\mathrm{H}$-free CSPN evolution without the born-again sequence of events. The AGB final thermal pulse (AFTP) scenario requires some mechanism to shed the remaining small envelope mass exactly after the final AGB thermal pulse with efficient dredge-up. In view of the physics uncertainties, in particular of envelope convection, this scenario is not inconceivable. De Marco et al. (2003b) and De Marco et al. (2003a) have tentatively investigated the spiraling-in and swallowing of a massive planet or very low mass stellar companion as an enhanced mass-loss process for the AFTP. The AFTP scenario is currently the most likely scenario for the hybrid CSPN (Napiwotzki et al. 1991).

Fig. 1 shows the comparison of different evolution channels. These calculations give an estimate of the difference of the tracks due to different surface chemistry and evolution history. In particular the highest temperature is the same for all channels.

More important for the location of the track in the HRD, and therefore for mass determinations using stellar-evolution tracks, is the progenitor evolution. Fig. 2 shows CSPN from various authors. The more recent $0.604 \mathrm{M}_{\odot}$ track is hotter than older tracks of the same mass. This difference is largely due to differences in the assumptions about AGB mass loss and the treatment of third dredge-up. As already shown by Blöcker (1995) CSPN with the same core mass can have significantly different tracks in the HRD. A core descending from an initially more massive progenitor simulated with a higher mass-loss rate will follow a hotter track than a core of the same mass with a lower-mass progenitor with lower mass loss. Similarly, efficient third dredge-up leads to a hotter CSPN track 


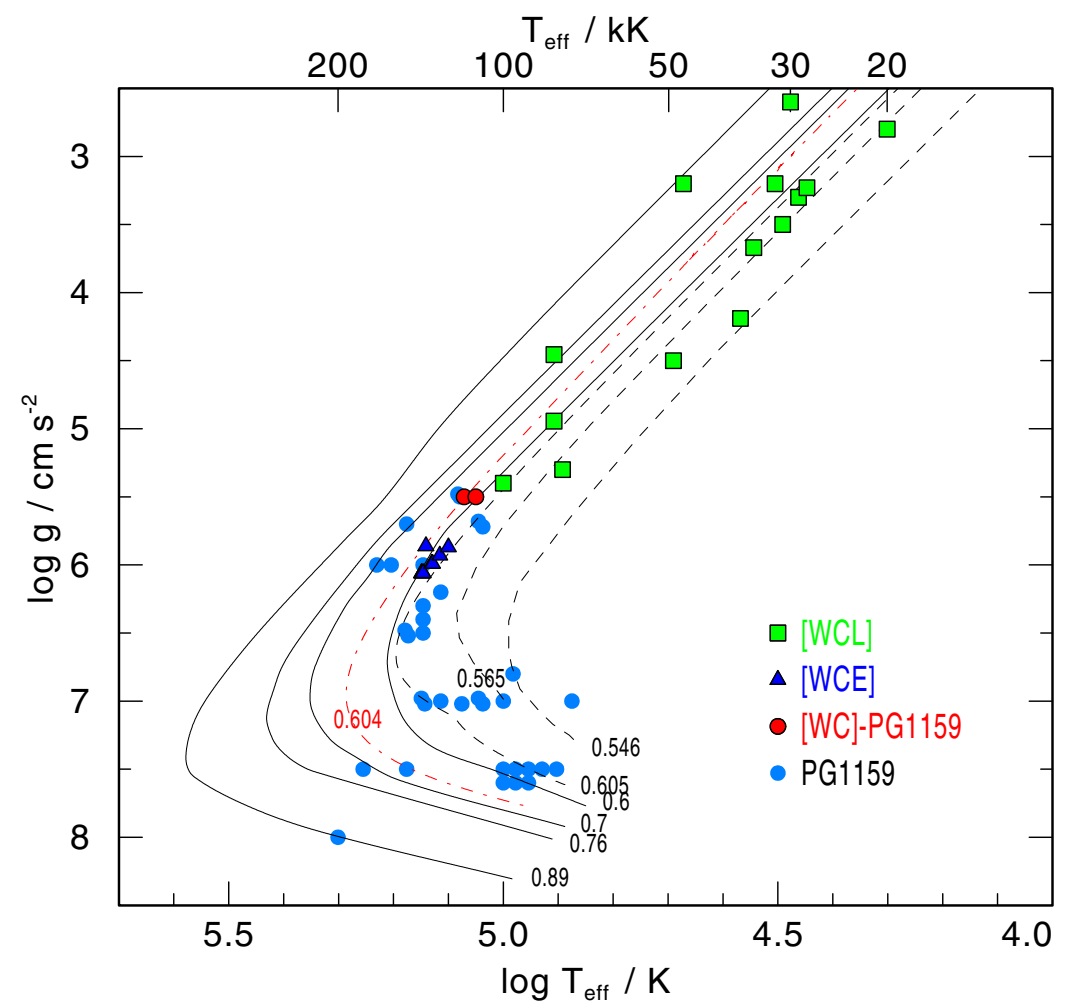

Figure 2. H-normal evolutionary tracks (labels: mass in $\mathrm{M}_{\odot}$ ) are from Schönberner (1983) and (Blöcker 1995) (dashed lines) and Wood \& Faulkner (1986). The $0.604 \mathrm{M}_{\odot}$ track Herwig (2003); Werner \& Herwig (2006) (dot-dashed line) is the final CSPN track following a VLTP evolution and therefore has H-deficient composition. Shown as well are hot hydrogen-deficient post-AGB stars in $g-T_{\text {eff }}$-plane. Wolf-Rayet central stars of early and late type ([WCE], [WCL], from Hamann (1997)), PG1159 stars as well as two [WC]-PG1159 transition objects (Abell 30 and 78) (see Werner \& Herwig 2006, for references) $T_{\text {eff }}$ for the [WC] stars is related to the stellar radius at $\tau_{\text {Ross }}=20$. This figure originally appeared in the Publications of the Astronomical Society of the Pacific (Werner \& Herwig 2006). Copyright 2005, Astronomical Society of the Pacific; reproduced with permission of the Editors.

compared with a core descending from a lower-mass progenitor with less third dredge-up. Binarity may influence the relation between core mass and HRD CSPN track additionally, through common envelope evolution, tidal synchronization, enhanced mass loss etc.

\section{Hydrodynamic simulations of He-shell flash convection}

We used the RAGE code to perform hydrodynamics simulations of a convection zone under conditions closely resembling those encountered in a typical He-flash during the hot pre-white dwarf evolution phase. The initial stratification is in hydrostatic equilibrium and consists of an entropy plateau, where the convection zone is expected to develop, with two adjacent stable regions. The convection is driven by a heat source in a thin layer at the bottom of the plateau to simulate the effect of He burning. A number of 2D and $3 \mathrm{D}$ runs with various resolutions and heating rates were produced. See Herwig et al. (2006) for more details.

The first panel in Fig. 3 shows the spatial entropy inhomogeneities at a snapshot during the onset of convection. Hot plumes rise from the bottom of the entropy plateau. They 

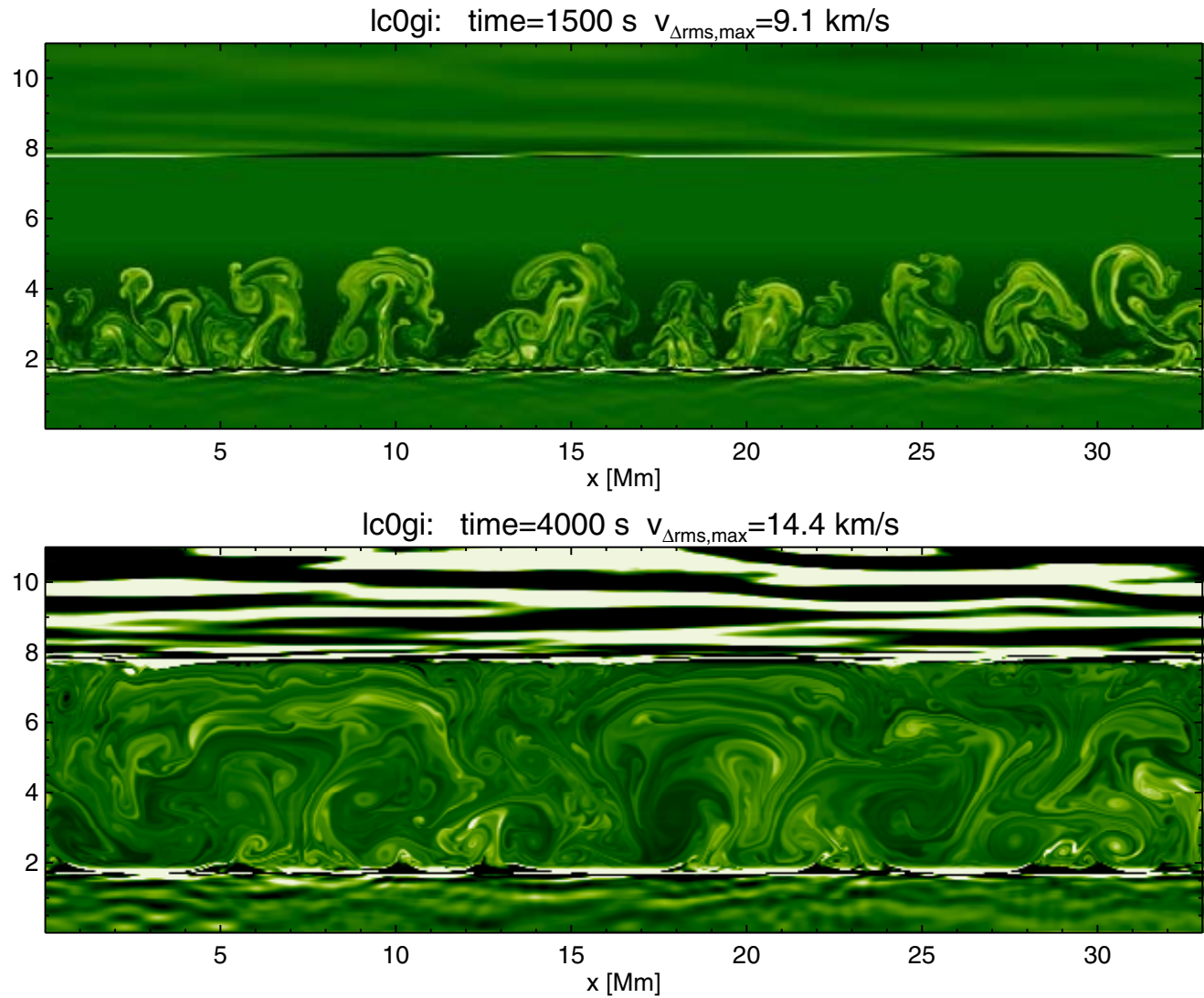

Ic0gi: time $=4050 \mathrm{~s} \mathrm{v}_{\Delta \mathrm{rms}, \max }=13.8 \mathrm{~km} / \mathrm{s}$

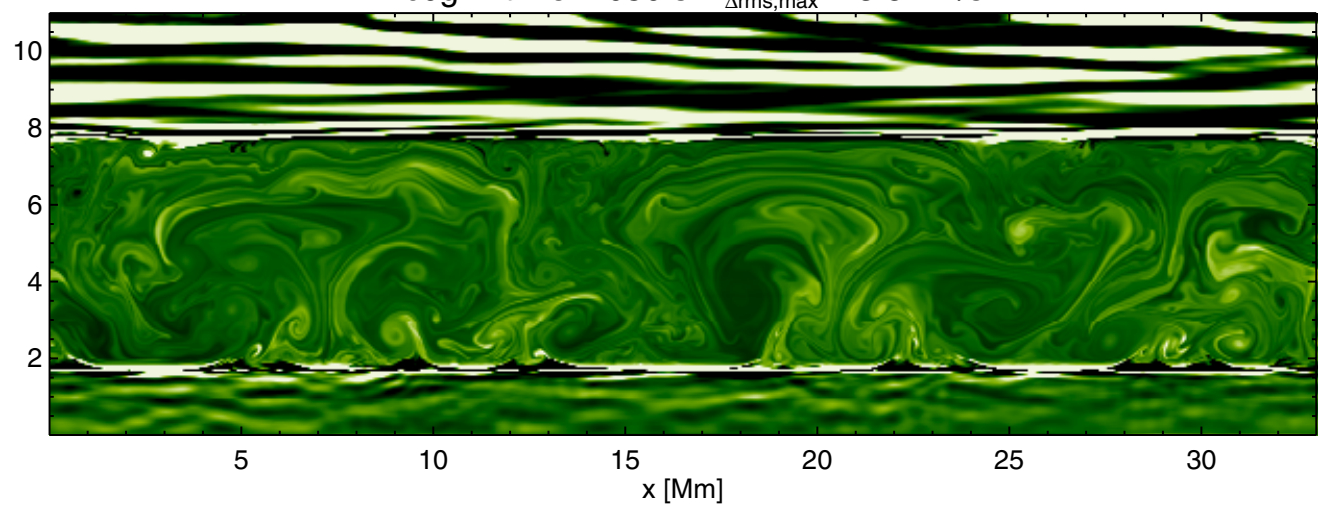

Figure 3. Entropy inhomogeneities for three snapshots of a $2 \mathrm{D}$ simulation with a resolution of $2400 \times 800$ grid points. The horizontal average of the entropy has been subtracted to render small fluctuations visible. A brighter color indicates material with an entropy excess (i.e. lower density than the surroundings). The overturning flow inside the convection zone clearly differs from the oscillatory motions due to gravity waves inside the stable layers.

merge while they grow in amplitude and vertical extent and start to excite gravity waves in the upper stable layers well before actually reaching the upper boundary. Later, there are typically four large instationary convective cells with a number of smaller eddies that continue to drive gravity waves in the adjacent layers (Press 1981). 


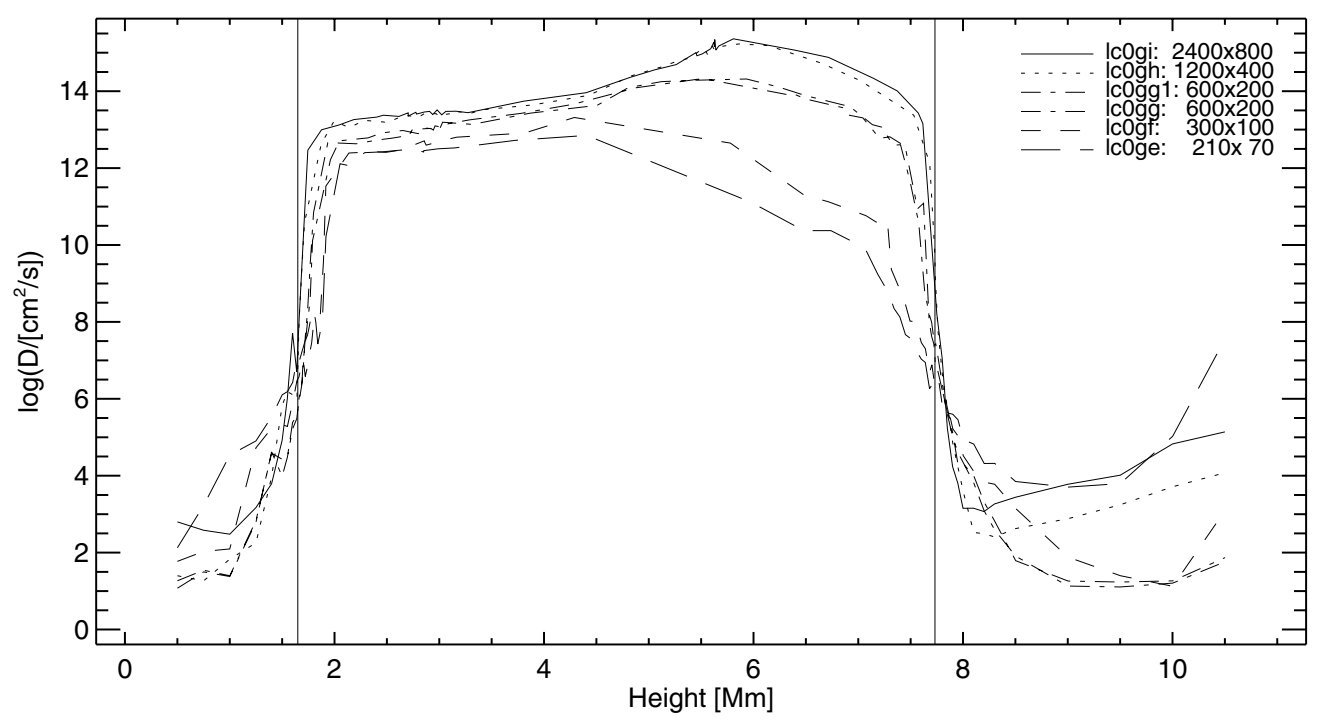

Figure 4. Logarithm of the diffusion coefficient versus height for models with different resolutions (indicated in the legend). The two vertical lines mark the boundaries of the entropy plateau at the start of the simulations. Later, they are slightly smeared out. The results from the runs with the two lowest resolutions differ significantly from the runs with next higher resolutions. Nevertheless, the agreement between the two highest-resolution runs is rather good.

The transition between convectively unstable and stable layers appears to be very sharp - without any plumes penetrating significantly into the the stable region. This is very different from the situation of thin stellar surface convection zones (Freytag et al. 1996). However, $k$ - $\omega$-diagrams for the vertical velocity at various height levels clearly show the mode signature of the gravity waves well inside the convection zone and also evidence of the convective flow reaching into the stable layers (Herwig et al. 2006).

Of particular interest are the mixing properties across the convection boundaries. The mixing is efficient inside the convection zone. Sometimes blobs of relatively hot material are sheared off the boundary to the top stable zone (see e.g. the tiny bright spots near the top left of the convection zone in the middle and bottom panels in Fig. 3) and are transported rapidly into deeper layers once they are entrained in a downflow region. Future simulations that include various species and nuclear reactions will show what happens if H-rich material is brought in this way into layers where it can burn.

The velocities in the stable layers are not much smaller than in the convection zone. However, wave motions are almost reversible and do not contribute much to the mixing. To quantify the mixing the trajectories of tracer particles (150 particles per horizontal layer in 68 layers) have been integrated based on snapshots with a high sampling rate of $0.5 \mathrm{~s}$. The spread of the relative vertical positions - or alternatively the relative entropy - of the particles in each ensemble with time gives a measure of the diffusion coefficient with height, plotted in Fig. 4 for models with different numerical resolutions. The large diffusion coefficient within the convection zone drops rapidly near the boundaries. This decay reaches slightly into the stable regions and can be described by one (at the top) or two (at the bottom) exponentials (Freytag et al. 1996).

The determination of the very small mixing coefficients far away from the convection zone is not very reliable. This small amount of mixing in the stable layer is due to internal gravity waves, and may be far reaching. 


\section{Conclusions}

Among the CSPN channels, the evolution leading to H-deficient surface composition is of great importance for constraining nucleosynthesis and mixing in the AGB progenitors. A clear correlation exists, e.g. between the mixing efficiency at the bottom of the He-shell flash convection and the $\mathrm{O}$ abundance in the intershell that is subsequently observed in H-deficient CSPN. AGB calculations with parameterized overshoot calculations by Herwig (2000) showed that more convective extra-mixing leads to higher O abundance, and that an exponential decay parameter for extra-mixing (see Herwig (2000) for the definition of $f \approx 0.01$ would be consistent with CSPN observed $\mathrm{O}$ abundances in the range $8-20 \%$ by mass. Our preliminary analysis of hydrodynamic simulations of the Heshell flash convection zone quantitatively agrees with this level of convective extra-mixing. We regard this agreement of hydrodynamic simulation and astrophysical observation as a successful validation of the computational simulations, and feel encouraged to continue this work towards more complex simulations of convective-reactive mixing.

\section{Acknowledgements}

This work was carried out in part under the auspices of the National Nuclear Security Administration of the U.S. Department of Energy at Los Alamos National Laboratory under Contract No. DE-AC52-06NA25396, and funded by NNSA's Advanced Simulation and Computing (ASC), Verification and Validation Program.

\section{References}

Blöcker, T. 1995, A\&A, 299, 755

Blöcker, T. 1999, in Asymptotic Giant Branch Stars, ed. T. Le Bertre, A. Lebre, and C. Waelkens, ASP, IAU Symp. 191, 21

Blöcker, T. 2001, APSS, 275, 1

Busso, M., Gallino, R., \& Wasserburg, G. J. 1999, ARA\&A, 37, 239

De Marco, O., Sandquist, E. L., Mac Low, M., Herwig, F., \& Taam, R. E. 2003a, in The Eighth Texas-Mexico Conference on Astrophysics: Energetics of Cosmic Plasmas, 24

De Marco, O., Sandquist, E. L., Mac Low, M.-M., Herwig, F., \& Taam, R. E. 2003b, in Revista Mexicana de Astronomia y Astrofisica Conference Series, 34

Freytag, B., Ludwig, H.-G., \& Steffen, M. 1996, A\&A, 313, 497

García-Berro, E. \& Iben, Jr., I. 1994, ApJ, 434, 306

Hamann, W.-R. 1997, in Planetary Nebulae, ed. H. J. Habing \& H. J. G. L. M. Lamers (Kluwer), IAU Symp. 180, 91

Herwig, F. 2000, A\&A, 360, 952

Herwig, F. 2001a, ApJ Lett., 554, L71

Herwig, F. 2001b, APSS, 275, 15

Herwig, F. 2003, in Planetary Nebulae: Their Evolution and Role in the Universe, ed. S. Kwok, M. Dopita, \& R. Sutherland, ASP, IAU Symp. 209, 111

Herwig, F. 2005, ARA\&A, 43, 435

Herwig, F., Blöcker, T., Langer, N., \& Driebe, T. 1999, A\&A, 349, L5

Herwig, F., Freytag, B., Hueckstaedt, R. M., \& Timmes, F. X. 2006, ApJ, 642, 1057

Iben, Jr., I., Kaler, J. B., Truran, J. W., \& Renzini, A. 1983, ApJ, 264, 605

Iben, Jr., I. \& Renzini, A. 1983, ARA\&A, 21, 271

Lattanzio, J. C. 1992, PASA, 10, 120

Lattanzio, J. C. \& Boothroyd, A. I. 1997, in Astrophysical Implications of the Laboratory Study of Presolar Materials, ed. T. Bernatowitz \& E. Zinner, AIP Conf. Ser., 402, 85

Lawlor, T. M. \& MacDonald, J. 2003, ApJ, 583, 913

Meyer, B. S. 1994, ARA\&A, 32, 153

Miller Bertolami, M. M., Althaus, L. G., Serenelli, A. M., \& Panei, J. A. 2006, A\&A, 449, 313 
Napiwotzki, R. 1999, A\&A, 350, 101

Napiwotzki, R., Schönberner, D., \& Weidemann, V. 1991, A\&A, 243, L5

Press, W. H. 1981, ApJ, 245, 286

Scalo, J. M., Despain, K. H., \& Ulrich, R. K. 1975, ApJ, 196, 805

Schönberner, D. 1983, ApJ, 708, 272

van Winckel, H. 2003, ARA\&A, 41, 391

Werner, K. \& Herwig, F. 2006, PASP, 118, 183

Willson, L. A. 2000, ARA\&A, 38, 573

Wood, P. R. \& Faulkner, D. J. 1986, ApJ, 307, 659 